\title{
Aprendizaje de la sostenibilidad basada en la cosmovisión andina amazónica ${ }^{1}$
}

\author{
Teresa Salinas Gamero \\ Universidad Ricardo Palma \\ teal33@yahoo.es
}

\begin{abstract}
RESUMEN
Esta investigación tiene tres aspectos relacionados a la innovación en Educación para el Desarrollo Sostenible (EDS): (i) cambios en los marcos filosóficos, epistemológicos y didácticos de la educación; (ii) el cuestionamiento de las nuevas funciones de la educación superior; y (iii) la evaluación e inclusión de una cosmovisión y racionalidad andina amazónica en los entornos universitarios. Uno de los resultados transformadores de esta experiencia fue promover una investigación educativa compleja, transdisciplinaria, fenomenológica y hermenéutica que promueva el diálogo intercultural. Se desarrolló en el marco del Diplomado de Biodiversidad y Saberes interculturales, realizado en la región San Martín, en las comunidades Quechua-Lamas, por el Instituto Peruano del Pensamiento Complejo Edgar Morin (IPCEM), con financiamiento de la cooperación alemana. Deutsche Gesellschaft für Internationale Zusammenarbeit (GIZ) GmbH y el Programa de las Naciones Unidas para el Ambiente - PNUMA. Además, contó con el apoyo del consejo académico regional constituido por organizaciones locales y el centro Waman Wasi. El Instituto para el Estudio Avanzado de la Sostenibilidad de la Universidad de las Naciones Unidas (UNU-IAS) reconoció esta experiencia con el RCE Award for Outstanding Flagship Project en la novena conferencia mundial de RCE, celebrada en 2014 en Okayama, Japón.
\end{abstract}

Palabras clave: Aprendizaje, complejidad, transdisciplinario, comunidades Quechua Lamas, visión intercultural, Educación para el Desarrollo Sostenible.

\section{Sustainability learning based on the Andean Amazonian Cosmo vision}

\begin{abstract}
This research has three aspects in relation to Education Innovation for the Sustainable Development: (i) changes in the philosophical, epistemological and didactic framework of education, (ii) the questioning of the new functions of the higher education and (iii) the evaluation and inclusion of an Andean Amazonian Cosmo vision and rationality in the University environment. One of the transformer results of this experience was to put into practice complex, transdisciplinary, phenomenological and hermeneutical education researches that promote an intercultural dialogue. It was developed in the «Diplomado en Biodiversidad y Saberes interculturales» (Diploma course in Biodiversity and Intercultural Knowledge) that took place in the San Martin region in the Quechua Lamas communities. It was organized by the Instituto Peruano del Pensamiento Complejo Edgar Morin -IPCEM (Peruvian Institute for Complex Thought Edgar Morin -IPCEM) with the German cooperation funding of the Deutsche Gesellschaft für Internationale Zusammenarbeit (GIZ) $\mathrm{GmbH}$ and the United Natiosn Environment Programme. It

1 Traducción del artículo de investigación publicado en Academia and Communities: Engaging for Change Innovation in Local and Global Learning Systems for Sustainability Learning Contributions of the Regional Centres of Expertise on Education for Sustainable Development, UNU-IAS, Tokyo, Japan, 2018
\end{abstract}


was also support by the academic regional council constituted by local organizations and the "Waman Wasi» centre. The UNU Institute for the Advanced Study of Sustainability (UNU-IAS) recognized this experience with the RCE Award for Outstanding Flagship Project in the 9th Global RCE Conference realized in 2014 in Okayama, Japan.

KeYwords: Learning, complex, transdisciplinary, Quechua Lamas communities, intercultural vision, Education for Sustainable Development

\section{Innovación en EDS}

Tres aspectos constituyen los puntos de partida: (i) cambios en los marcos filosóficos, epistemológicos y didácticos de la educación; (ii) el cuestionamiento de las nuevas funciones de la educación superior y (iii) la evaluación e inclusión de una cosmovisión y racionalidad andino-amazónica en los entornos universitarios. Para comenzar el Diplomado en Biodiversidad y Diálogo de Saberes Interculturales, se requirió que la Universidad Ricardo Palma validara el conocimiento de lo andino-amazónico. Esto provocó un intenso debate. Finalmente, se nombró un consejo académico regional para validar tanto a los líderes indígenas que participarían cumpliendo la función de maestros, como a los que asistirían en calidad de estudiantes. Estos últimos, al llevar el programa, fueron educados en el uso de las TIC y el aula virtual para poder comunicarse a nivel local y nacional. Aunque esto requirió un aumento del $30 \%$ en el tiempo del programa del diplomado, los estudiantes luego pudieron asistir a conferencias y debates regionales, y presentar sus propuestas.

\section{Transformación social}

A nivel local, el diplomado validó el conocimiento tradicional y fortaleció a los líderes de las comunidades Quechua Lamas que asistieron. Los líderes de las comunidades indígenas, maestros, técnicos y profesionales de instituciones privadas y públicas, así como los gobiernos locales, fortalecieron su visión intercultural y establecieron la necesidad de desarrollar visiones y acciones compartidas que valoren el conocimiento académico y el conocimiento ancestral relacionado con la regeneración de la biodiversidad y su sostenibilidad.

\section{Implicaciones para el Desarrollo de Instituciones de Conocimiento}

Uno de los resultados transformadores de esta experiencia fue poner en práctica una investigación educativa compleja, transdisciplinar, fenomenológica y hermenéutica que promovieran un diálogo de saberes entre los agentes sociales de los diferentes niveles de realidad. Todo ello se ha fortalecido en la universidad y se ha extendido a otros proyectos. A nivel nacional, se consolidó un grupo de trabajo para la innovación educativa en la Universidad Ricardo Palma. A nivel mundial, se promovió la creación de redes y se compartieron contribuciones y buenas prácticas en los eventos organizados por GIZ GmbH, PNUMA y UNU. 


\section{Deshumanización del conocimiento «científico»}

A mediados de la década de 1970, se hizo evidente una nueva «revolución científicotécnica» mundial. Esta se caracterizó por una sinergia entre la mecánica cuántica, la informática y la biología molecular, y por desarrollos sin precedentes en los niveles de información a nivel mundial. Estas transformaciones han permeado todas las actividades humanas y aumentado la complejidad del conocimiento y de la vida en el planeta.

Influenciada por las ideas de Descartes y Newton, la cultura occidental construyó el conocimiento al buscar el orden, descartando las contradicciones, fragmentando las totalidades, reduciendo y rechazando la incertidumbre en los procesos emergentes; en definitiva, construyó el conocimiento mediante el paradigma de la simplificación. Si bien esta forma de conocimiento ha traído aspectos positivos (en medicina y tecnología, etc), sus consecuencias han sido perjudiciales para los modelos mentales de la humanidad. De hecho, las acciones que siguieron finalmente han puesto en peligro la vida en la Tierra.

El pensamiento positivista y reduccionista, reforzado por la razón instrumental y la mercantilización de la vida, bien puede ser considerado responsable de la acción depredadora de los humanos sobre la naturaleza y de la ceguera para comprender y abordar los graves problemas de la humanidad. Esta comprensión estrecha del mundo aceptó solo un método para crear conocimiento científico, y al mismo tiempo invalidó otros sistemas de conocimiento. Históricamente, con la conquista de las Américas, esta forma de pensar sobre el mundo se impuso en las tierras conquistadas; al mismo tiempo, los métodos indígenas de construcción de conocimiento, así como las prácticas indígenas sociales y espirituales, fueron brutalmente reprimidas. Según Ramos (1992)

La pérdida de referencias culturales propias hace que las personas entren en ese sistema arbitrario de categorías por el cual 'desarrollado' son aquellos que consumen más energía y los 'salvajes' que [consumen] menos. Este tipo de matriz cultural, en la que estamos cautivos, crea un circuito de valores alienados con respecto a los tipos de conocimiento y cambia el orden, la esencia y las características (por el pequeño tamaño de nuestro mercado y su relación dependiente) de contenidos y formas del conocimiento. (p. 33)

Un mayor desarrollo de las ciencias hizo evidente que algunas de las propiedades emergentes de la vida y la naturaleza no pueden ser abordadas necesariamente por la ciencia determinista y reversible. Según la ciencia clásica, la masa y la energía son las fuentes de cambio y transformación. El desarrollo de la biología condujo a una mejor apreciación de cómo se organiza la vida y cómo emergen ciertos procesos (como la conciencia humana o el cambio climático). La información aparece también como otra fuente de cambio y transformación. 
Tales entendimientos, que han venido evolucionando, han producido cambios en las formas de pensar y vivir en el mundo, las estructuras de conocimiento y la forma de hacer ciencia. Fomentan el auge de las ciencias de la complejidad y el pensamiento complejo (Morin, 1995) y las estructuras disipativas de la termodinámica de no equilibrio (Prigogine y Stengers 1983), entre otras, que han integrado la irreversibilidad del mundo real en la ciencia.

Esta "revolución científico-técnica» global abrió nuevos marcos epistémicos para comprender los fenómenos naturales y sociales que subyacen en la existencia humana. En el nuevo orden epistémico y social, los habitantes de los andes centrales del Perú todavía conservan su cultura. Existe la esperanza de que investigaciones sobre nuevos marcos epistemológicos de la ciencia que puedan respaldar nuevas formas de pensar, entender la condición humana, cuidar la biodiversidad y preservar la vida en la Tierra de modo tal que se conduzca a un cambio en las prácticas universitarias, lo cual provoca que se incluyan otros sistemas de conocimiento. Brevemente, lo que se necesita es una educación contextualizada y pertinente para alcanzar el desarrollo sostenible. Según Morin:

Es esencial, al unísono, globalizar y desglobalizar, crecer y disminuir, desarrollarse e involucrarse, conservar y transformar La globalización y la desglobalización tal como está orientada significa que si multiplicáramos los procesos culturales de comunicación y globalización, si fuéramos a crear una conciencia Madre-Tierra, una comunidad de conciencia del destino, también es necesario fomentar el desarrollo local dentro de la global. (Morin, 2011, p. 35)

\section{Cuatro desafíos para la educación superior en el Perú}

El sistema de educación superior en Perú fue creado por conquistadores con el objetivo de mantener el estado colonial, imponer su cultura y destruir la cultura indígena. Aunque la universidad ha sido una fuerza democratizadora desde el final de la era colonial, todavía mantiene su carácter colonizador en relación con la producción y la aplicación del conocimiento. Por eso, el trabajo de la universidad se basa en la repetición del conocimiento desarrollado en otros países y otros contextos, uno que no aborda las necesidades básicas de la población multicultural y multiétnica del Perú. Es urgente trabajar por la descolonización del conocimento y de la universidad peruana

Por ejemplo, los ingenieros y tecnólogos a cargo de la conservación de los ecosistemas en los Andes centrales del Perú tienen una tendencia a subestimar o ignorar el conocimiento ancestral y priorizar las lecciones que aprendieron en las aulas universitarias, aunque la mayoría de las veces estas pueden no estar adecuadamente contextuali- 
zadas. Este es un obstáculo importante para comprender mejor las realidades locales y culturales. Por lo tanto, dificulta la integración creativa y productiva del conocimiento para promover la protección de la biodiversidad y el desarrollo sostenible.

La educación en el Perú ha olvidado, en su mayor parte, la esencia del ser, la condición humana y la reflexión sobre quiénes son los peruanos. Las tensiones y contradicciones de la vida local y nacional también se reflejan en desafíos para el diálogo de saberes y constituyen la causa raíz de la crisis educativa. Además, determinan la falta de pertinencia y de calidad en la educación. Sin embargo, estos problemas no se abordan. Incluso, cuando se han hecho algunos esfuerzos para tratarlos, las deficiencias continúan. La mayoría de los desafíos que forman parte de esa realidad son multidimensionales y recursivos; no obstante, algunos de ellos, debido a sus impactos significativos, no deben pasarse por alto.

El primer desafío tiene que ver con las estructuras de conocimiento, es decir, cómo las personas construyen el conocimiento, cómo el conocimiento construye a las personas y cómo el conocimiento construye los patrones de la mente. Es decir, se trata de saber si estos procesos ayudan a las personas a comprender el mundo, la dinámica de la vida, la biosfera y la naturaleza humana, y, sobre todo, si ayudan a desarrollar conocimiento pertinente.

El segundo tiene que ver con la visión reduccionista y fragmentada del sistema educativo. La educación no debe reducirse a áreas formales. El apoyo real y sistemático a esta, tanto si es formal como si no, es esencial y debe realizarse junto con políticas públicas sólidas para asegurar que los sectores privado y público (especialmente los medios de comunicación), y la sociedad civil cumplan con la responsabilidad social. También es esencial garantizar la implementación de espacios públicos de aprendizaje y centros de conocimiento interactivos como medios efectivos para aprender a lo largo de toda la vida a través de la Educación para el Desarrollo Sostenible (EDS).

Un tercer reto es la mercantilización de la educación. Al permitir que la educación sirva al mercado, su esencia como un derecho inherente a la condición humana se ha distorsionado. La privatización masiva de la educación no ha mejorado su calidad, ni sus valores éticos y morales. Por ello, se puede afirmar que todavía está pendiente una evaluación de la educación privada en el Perú.

Finalmente, se debe considerar la no pertinencia de la educación, puesto que no prepara a los ciudadanos para enfrentar las incertidumbres de la vida ni para abordar los graves problemas de la humanidad (pobreza, violencia, delincuencia, hambre, cambio climático, etc.). 


\section{Estableciendo estudios de sostenibilidad.}

\section{Repensando la Universidad a la luz de una tradición de pensamiento diferente}

Los graves desafíos que enfrenta la humanidad nos llevan a cuestionar el papel que juega la educación en la construcción del mundo. ¿Cómo esta contribuye a moldear las estructuras mentales de la humanidad? ¿Por qué es tan precaria la creación de conocimiento pertinente en las universidades? ¿Qué marcos filosóficos o epistemológicos podrían sustentar la EDS? ¿¿Desde qué marco epistemológico podría la universidad comprender y validar los conocimientos de una comunidad indígena? ¿Cómo han cuidado las comunidades indígenas de los Andes centrales la biodiversidad durante miles de años? ¿Qué temas son de interés mutuo tanto para las universidades como para las comunidades indígenas?

El desafío fundamental para la EDS radica en la forma de pensar y estar en el mundo, en el nivel de conciencia para comprender cómo los humanos están destruyendo el planeta, y en el compromiso de transformarlo. Esto requiere repensar el papel de las personas como individuos y seres sociales, así como la relación de la humanidad con la naturaleza. Esto significa repensar la educación, transformar los fundamentos de la educación y los procesos de aprendizaje y, por lo tanto, «volver a encantar» el significado de la vida en el planeta.

Las personas necesitan transformar los modelos mentales lineales y fragmentados, basados en la razón instrumental, en una razón ética y humanista, es decir, en una educación que ayude a regenerar la vida y hacer más feliz a la humanidad.

Cuadro 1: La propuesta Ayni

La propuesta Ayni constituye la búsqueda continua para alentar el cambio de la visión lineal y fragmentada de los sistemas educativos (basada en Salinas, 2009). Esta propuesta presenta una visión dinámica compleja, transdisciplinar, hermenéutica y fenomenología del Sistema de Educación Superior. En esta propuesta, solo se incluyen algunas variables e interacciones; por lo tanto, puede considerarse como un tipo de reduccionismo. Sin embargo, podría jugar un papel clave para crear visiones compartidas de los procesos.

Ayni concibe los procesos de la educación en bucles recursivos que interactúan con los sistemas sociales y naturales, en un proceso de auto-eco-organización ${ }^{2}$. Estas inter y retroacciones van transformando la organización y por ende las estructuras mentales y regula las formas de traducir la realidad y la intersubjetividad, tanto individual como colectivamente, así como las relaciones con el entorno. Todos estos procesos se desarrollan de forma simultánea, realizando funciones locales y globales. De esa manera, las emergencias surgen y proporcionan retroalimentación a este mismo sistema, a los seres humanos (individual y colectivamente) y a los ecosistemas. Esta dinámica determina la calidad de vida y de la educación, y la relevancia y contextualización de esta última.

2 La autoorganización nos permite considerar una evolución creativa que integra y transforma las capacidades de orden, desorden, y la organización (ecológica, biofísica y cósmica) en las capacidades psico-cerebrales que organizan el conocimiento. 


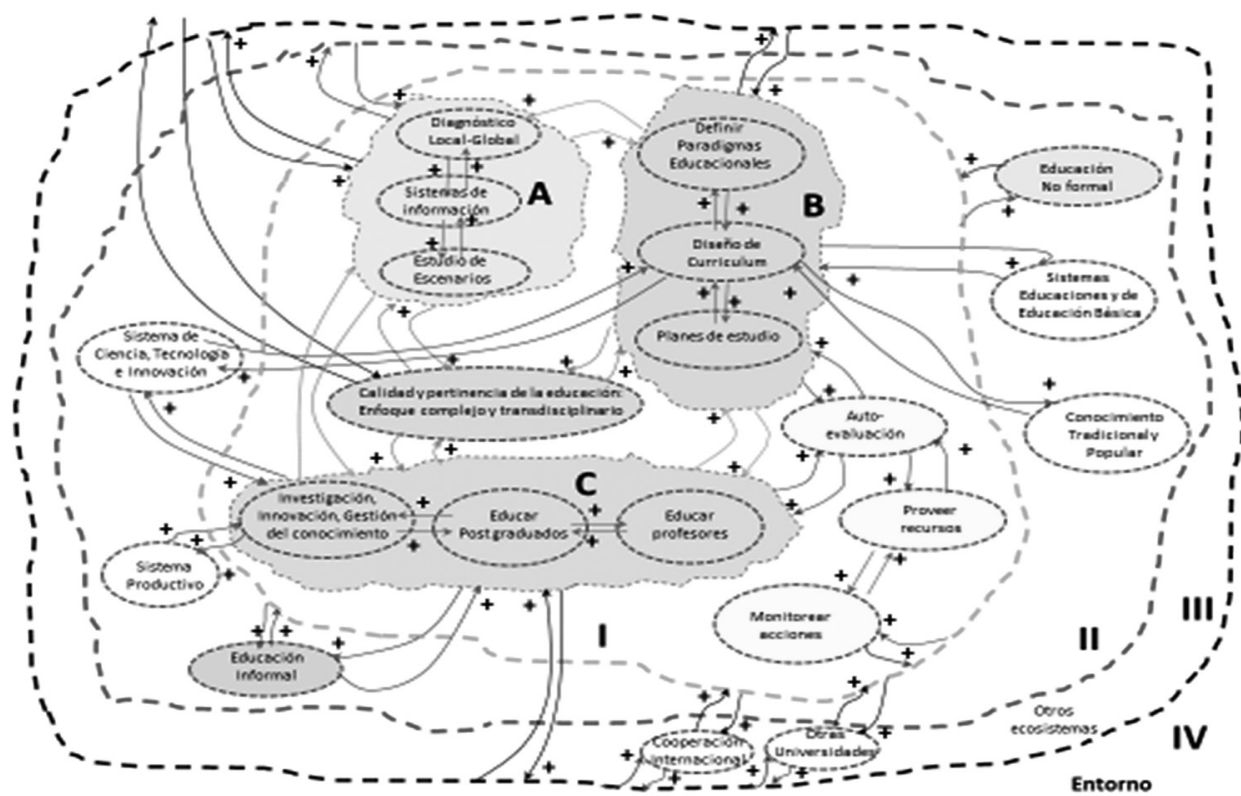

Figura 1: Propuesta Ayni para la educación superior. Nivel I: Universidad; Nivel II: Nacional; Nivel III: Global (Ver Apéndice A).

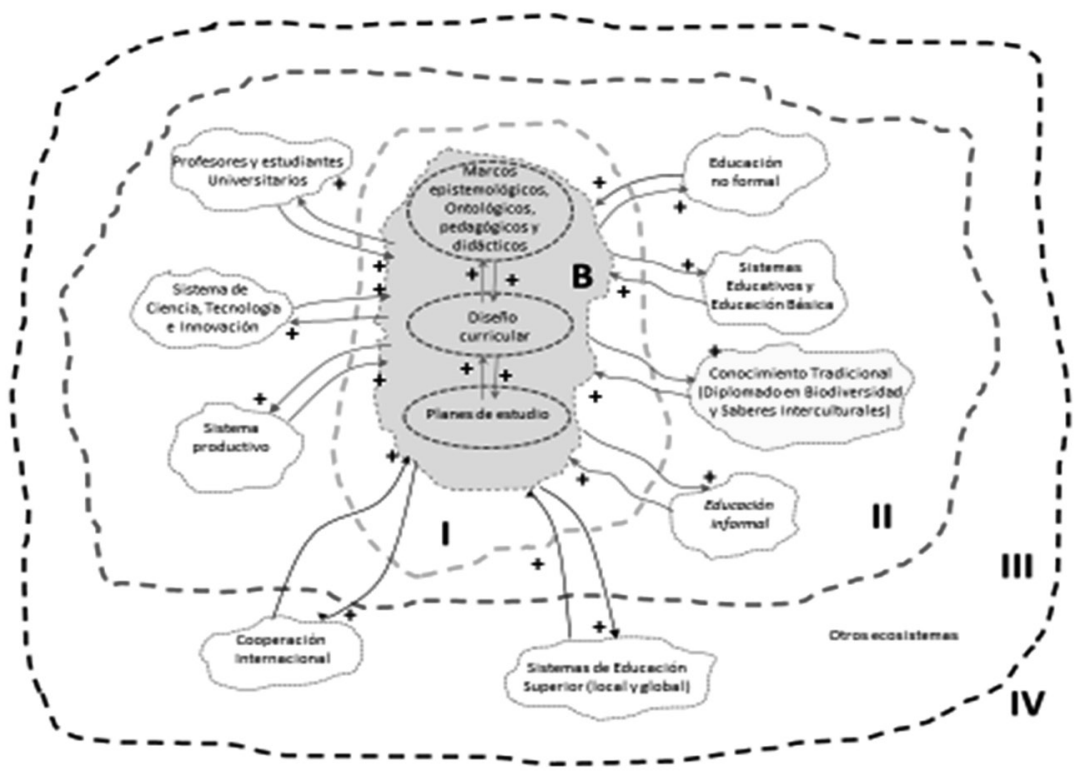

Entorno

Figura 2: propuesta de Ayni para la educación superior. Nodo B: Interacciones para un currículo pertinente y contextualizado (Ver Apéndice A). 
Desde la perspectiva del sistema de educación superior y las ideas de Morin, Prigogine, Maturana, Nicolescu y algunos otros, la propuesta Ayni (véanse el cuadro 1, la figura 1 y la figura 2) indaga otra visión para explorar la transformación del aprendizaje de lineal a sistémico en toda su complejidad. Además, valida otras formas de generar conocimiento y planes de estudios relevantes y contextualizados.

Ahora bien, recordemos que, según Maturana (2014):

Educar es crear, realizar y validar en la convivencia, una forma particular de convivencia. Esto siempre se hace en una red de charlas que coordina el hacer y la emoción de los participantes... esto significa dar a cada ciudadano elementos para una tarea autónoma, social y ecológicamente responsable (Maturana, 2014, p. 147).

Para que esto sea posible, la educación debe centrarse en el amor, el respeto mutuo y la confianza. Maturana también señaló que «el conocimiento adquirido debería tener algún sentido en el entorno vital en el que viven los estudiantes» (ibid., 148).

El enfoque intercultural de la educación es fundamental aquí. Se propone la imposibilidad de comprender toda la experiencia humana desde una sola perspectiva. Perú necesita una educación cuyos planes curriculares sean pertinentes y contextualizados, que pueda responder a los desafíos locales, nacionales, globales, y contribuya a resolver las necesidades básicas de la población; es decir, a superar la miseria económica, cultural y espiritual.

No basta con seguir la lógica de una sola cultura. Otros métodos complementarios pueden ayudar a reorientar la educación. En este sentido, Prigogine y Stengers (1983, 279) dijeron:

La metamorfosis de las ciencias contemporáneas no es una ruptura. Creemos, por el contrario, que nos lleva a comprender el significado y la inteligencia de los antiguos conocimientos y prácticas que la ciencia moderna orientada hacia el modelo de fabricación técnica automatizada había dejado de lado.

Los Andes centrales de América del Sur albergan una herencia milenaria de conocimientos y prácticas sociales (Recuadro 2) que se han ocupado de los complejos y frágiles ecosistemas andinos. Diferentes frases caracterizan la forma de ser y vivir en las comunidades indígenas. Las comunidades de la Amazonía Andina de habla quechua lo llaman sumak kausay ('buen vivir' o 'vivir bonito' en español, 'the good way of living' en inglés). 
CuAdro 2: La cosmovisión andino-amazónica

El Perú es rico en diversidad biológica y cultural. La Amazonía peruana representa el 60\% del territorio peruano. Aunque la región San Martín representa solo el 6,6\% del territorio amazónico, es la que presenta mayor densidad de población en la Amazonía, hogar de aproximadamente 800,000 personas indígenas y no indígenas, que representan el 30\% de la población amazónica total.

Esta realidad, causada en gran medida por la inmigración andina, ejerce presión sobre la naturaleza en la medida en que algunos lugares, como el Alto Mayo, se encuentran entre los más degradados del país. Perú tiene 42 grupos étnicos amazónicos con una población de 340,000. Tres están en la región de San Martín: Awajún o Aguaruna, Quechua-Lamas y Chayahuitas. Awajún y Quechua-Lamas son el segundo y tercer grupo étnico más grande en el Amazonas después de los Ashaninka. Estas personas tienen un profundo conocimiento de su territorio, y han mantenido y preservado, hasta ahora, no solo su conocimiento, sino también sus valores y prácticas para cuidar la naturaleza.

La cosmovisión andino-amazónica tiene una comprensión sistémica de las personas y la naturaleza. Evidenciada en la vida cotidiana de las personas y en la educación indígena, concibe la interrelación fundamental hasta el punto de que su pensamiento recursivo es obvio. En ella, uno hace conexiones y tiene un diálogo incesante con sí mismo y con todo lo que existe en la naturaleza. El principio de «reciprocidad» gobierna cualquier actividad y toda interacción entre el ser humano y la naturaleza.

Antonio Peńa Cabrera destacó algunas características de la racionalidad andino-amazónica:

La persona andina amazónica... se inclina hacia la diversificación y la variedad, no solo respeta la pluralidad existente, sino que también la enriquece... La gente andina amazónica tenía una conciencia ecológica, basada en un sentido de responsabilidad colectiva y una percepción del orden cósmico. No ha habido ninguna agricultura significativa en ningún lugar de la Tierra de más de 3000 metros de altura, excepto en los Andes. La racionalidad que hizo posible la vida y la diversidad en una altitud tan alta fue la andina amazónica (Peña Cabrera 1998, 16-19).

Para sobrevivir, los andinos necesitan el colectivo. Como lo explicó un líder de la comunidad indígena Quechua-Lamas (apu en idioma Quechua), practican choba-choba (asistencia mutua). Así lo manifiestan sus palabras: «Lo que puede hacer en una semana, puede tomar un día para hacerlo juntos; nuestra comunidad y familia en su totalidad contribuirían; además, todos juntos comeríamos, bailaríamos y agradeceríamos a la madre naturaleza».

¿Cómo recuperar estos conocimientos para una educación intercultural? ¿Qué piensan los indígenas al respecto? Para ingresar a una comunidad indígena, uno tiene que cumplir con una serie de protocolos establecidos. Para ellos, la confianza mutua es primordial. Los indígenas sufrieron y sufren muchos engaños, lo que tuvo un impacto negativo en su visión del mundo y sus costumbres, y animó a sus jóvenes a emigrar a las ciudades. En este caso, a través del Consejo Académico de la región, se ha emprendido un trabajo con ellos para fortalecer la confianza mutua.

Con el objetivo de contribuir a recuperar el conocimiento indígena tradicional y los valores de la comunidad, es necesario fortalecerlos, hacerlos visibles y equipar a los maestros de escuela, autoridades, estudiantes y a la sociedad en general con una visión intercultural para la descolonización del conocimiento. Esto permitiría la validación e incorporación del conocimiento relevante de la Amazonía Andina en todos los niveles 
de los currículos educativos y en todos los lugares del territorio peruano. Además, los desafíos de repensar la educación son asumidos normalmente por las universidades. Según el marco legislativo actual, estos centros de estudios suelen cumplir tres funciones: formación académica, proyección social (extensión universitaria a la sociedad) e investigación. En opinión de la autora, estas funciones son insuficientes para lograr el desarrollo sostenible. La figura 3 muestra algunas funciones nuevas que deben incorporarse en la educación superior: administrar el diálogo de saberes, alentar la creación de conocimiento pertinente y promover la educación intercultural. La producción de conocimiento debe ser una de las principales funciones de las universidades.

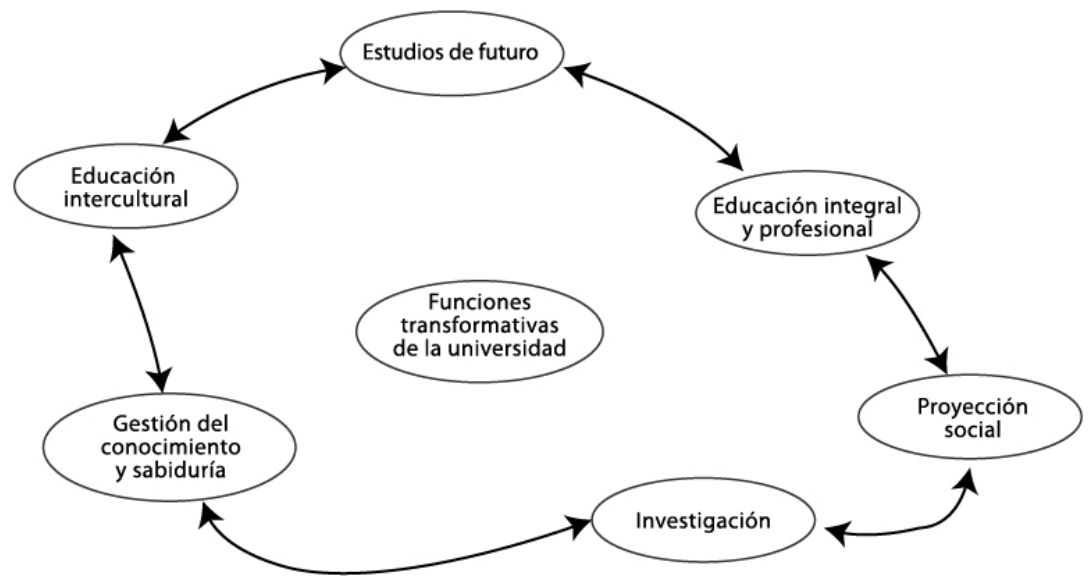

Figura 3. Nuevas funciones de la universidad para posibilitar el desarrollo sostenible.

Consciente de su responsabilidad social hacia la preservación de la diversidad biológica y cultural del Perú, el Instituto Peruano del Pensamiento Complejo Edgar Morin (IPCEM) de la Universidad Ricardo Palma, realizó el Diplomado en Biodiversidad y Diálogo Intercultural. El propósito fue conectar la tecnociencia y el conocimiento tradicional para la transferencia mutua de conocimiento y buenas prácticas, de manera que contribuyan con las EDS, capaces de generar soluciones a los desafíos locales y globales.

Es pertinente señalar aquí que este curso, que combina el trabajo académico con el desarrollo de las comunidades indígenas Quechua-Lamas de la región San Martín, se desarrolló como parte de las actividades del RCE Lima-Callao. La Universidad Ricardo Palma (URP), en asociación con otras organizaciones públicas y de la sociedad civil, creó este Centro Regional de Expertise (RCE) sobre Educación para el Desarrollo Sostenible, que fue reconocido por la Universidad de las Naciones Unidas en 2011 como parte de la Red Global RCE (Fadeeva et al. 2014). Por su parte, es el Instituto Peruano del Pensamiento Complejo Edgar Morin el que coordina el RCE Lima-Callao. 


\section{Fundamentos epistémicos del concepto de proyecto}

Las universidades peruanas solo reconocen el sistema de conocimiento occidental como válido. Sin embargo, para que la EDS sea posible, deben considerarse todos los sectores sociales, lo que genera que sea esencial investigar nuevos enfoques epistemológicos que respalden una educación capaz de integrar todas las visiones del mundo que coexisten en un territorio determinado.

El debate sobre el enfoque epistemológico en el grupo de investigación del RCE Lima-Callao se basa en las propuestas de Morin, Prigogyne, Maturana y Nicolescu, entre otras. La teoría de Morin cuestiona el pensamiento fragmentador y reductivo, basado en verdades absolutas, ciego a la comprensión de la dinámica de la vida. Asimismo, propone un pensamiento capaz de comprender la complejidad de los fenómenos naturales y sociales, algunas de sus características son : el principio dialógico, el principio de recursividad organizacional y el principio hologramático (Morin, 1981). El primero permite asumir racionalmente la inseparabilidad de las nociones contradictorias para concebir el mismo fenómeno complejo. Eso sucede, por ejemplo con las nociones antagónicas de la vida y la muerte o el bien y el mal, que coexisten en la misma realidad. El segundo explica cómo los efectos o productos son causa y efecto de un mismo proceso. Esta noción requiere que uno considere los procesos de autoorganización y autoproducción que caracterizan la complejidad de los fenómenos naturales y sociales, especialmente, la educación. Por último, el principio hologramático afirma que la parte no solo es la parte en el todo, sino que el todo está en la parte; en última instancia, trasciende tanto el reduccionismo, que ve solo la parte, como el holismo, que ve solo el todo.

Si la educación se lleva a cabo en una red de intercambios que coordina las acciones y las emociones de las personas involucradas, existe la necesidad de fomentar reflexiones en la educación que nos hagan conscientes de las limitaciones que nosotros, los humanos, tenemos para comprender las dinámicas complejas del universo, la Tierra, el cuerpo, la mente y el espíritu, así como del significado de la cultura.

En este proyecto, los siete saberes de Edgar Morin sobre la educación para el futuro son el punto de partida para la investigación. Estos son los siguientes:

1) La ceguera del conocimiento: error e ilusión («preparar a la mente para enfrentar la amenaza constante de error e ilusión»);

2) Los principios del conocimiento pertinente (la necesidad de ubicar el conocimiento parcial dentro de la complejidad de su realidad y la totalidad de su contexto);

3) Enseñar la condición humana (clave para la educación, los seres humanos son simultáneamente seres físicos, biológicos, psicológicos, culturales, sociales e históricos); 
4) Enseñar la identidad de la Tierra (conciencia de la complejidad de las crisis globales, reconocimiento del destino compartido de la humanidad);

5) Enfrentar las incertidumbres («la educación debe incluir el estudio de las incertidumbres que han surgido en las ciencias físicas —-microfísica, termodinámica y cosmología-, las ciencias de la evolución biológica y las ciencias históricas»);

6) Entenderse mutuamente (que está ausente de la educación, a pesar del hecho de que es vital para las relaciones humanas y «que es una necesidad vital para llevar las relaciones humanas más allá de su bárbara etapa de malentendidos»); y

7) La ética de la humanidad (un ser humano es al mismo tiempo un individuo, un miembro de una sociedad y un miembro de una especie). (Morin, 1999, pp. 1-4)

Según el mismo autor, a partir de este momento pueden observarse los dos grandes objetivos ético-políticos de este nuevo milenio: primero, establecer a través de la democracia una relación mutuamente controlada entre la sociedad y los individuos; segundo, pensar en la humanidad como una comunidad planetaria y así trabajar por una ciudadanía planetaria.

La perspectiva transdisciplinaria propuesta por $\mathrm{Nicolescu}^{3}$ se basa en una apertura educativa multidimensional y multirreferencial, que «revaloriza el papel de la intuición, el imaginario, la sensibilidad y el cuerpo en la transmisión del conocimiento» (Nicolescu, 1996). Esto permitiría el movimiento a través de diferentes niveles de realidad del objeto (micro, meso y macrocosmos) y diferentes niveles de percepción del sujeto (individuo, sociedad, etc.).

Implica aprehender (de otra manera y con otra estrategia) las disciplinas científcas y los conocimientos tradicionales y populares de diferentes culturas a través de un intercambio transdisciplinario de diálogo de saberes, que requiere nuevos enfoques y actitudes (reciprocidadr, respeto, tolerancia). De esa manera, se permite el surgimiento de nuevos conocimientos que no pueden obtenerse a partir de la linealidad, la simplificación y la reducción.

La epistemología es un examen de los orígenes, la naturaleza, los métodos y los límites del conocimiento... sin embargo, cualquier epistemología se basa en la tradición intelectual occidental». Desde este punto de vista: «Proponemos por tanto una comprensión más amplia de la epistemología, una que no la reduzca a una teoría del

3 Principios de transdisciplinariedad: 1) el axioma ontológico: Existen, en la naturaleza y en la sociedad, y en el conocimiento de la naturaleza y la sociedad, diferentes niveles de realidad del objeto y, en consecuencia, diferentes niveles de realidad del sujeto; 2) el axioma lógico: el paso de un nivel de realidad a otro está asegurado por la lógica del medio incluido; 3) el axioma de complejidad: la estructura de la totalidad de los niveles de realidad o percepción es una estructura compleja; cada nivel es lo que es porque todos los niveles existen al mismo tiempo (Nicolescu 1994). Sin embargo, la comprensión puede mejorarse a través de otros "conocimiento» como aquel sobre la tecnociencia, el tradicional y popular, y las experiencias de vida. 
conocimiento científico, sino más bien a una que abarque el espectro de vías de acceso al conocimiento y facilite el flujo de la vida. En este contexto, la epistemología podría entenderse como la reflexión sobre las condiciones de posibilidad de un conocimiento riguroso que apoya el acompańamiento de un grupo culturalmente distinguible por alguien que acompaña el aprendizaje mutuo (Ishizawa 2009, 9).

Desde el enfoque de educación comunitaria de las comunidades andino-amazónicas, la sabiduría nativa de los valores, las prácticas y el conocimiento tienen el potencial de proponer la naturaleza transdisciplinaria del diálogo de saberes. El 'buen vivir' de estas comunidades indígenas no es una receta, sino un despliegue intercultural constante. De acuerdo con Lajo, es el equilibrio entre sentirse bien (allin munay en quechua) y pensar bien (allin yachay) lo que resulta en hacerlo bien (allin ruay) para lograr la armonía (Lajo, 2005).

El buen vivir se basa en los principios de (a) la interconexión, o las dinámicas interconectadas de la vida, que no solo son aspectos relacionadas con el patrimonio humano, sino también aquellos producidos por un diálogo con todo lo que existe: ríos, animales, chacra, montańa, luna, deidades; (b) la reciprocidad (ayni en quechua), según la cual los miembros de todas las comunidades tienen algo que dar y necesitan algo para existir; y (c) la complementariedad, en la que todos cumplen una función de armonía, equilibrio, vivir en paz y comunidad. La vida nace en esa interacción de protección, respeto mutuo, amor, afecto, confianza, alegría y la danza con la naturaleza. De estas interacciones emergen algunas prácticas comunitarias como el choba-choba (palabra quechua que significa «ayuda mutua») o mikunas (un tipo de ritual de la comida). Según Aníbal Quijano:

Somos testigos del surgimiento de una nueva identidad histórica ... cuyo desarrollo podría producir una nueva existencia social liberada de la dominación, la explotación y la violencia ... Desde esta perspectiva, la propuesta de «vivir bien» es necesariamente una cuestión histórica abierta que debe investigarse, debatirse y continuamente practicarse (Quijano, 2015, p. 859).

Estos enfoques epistemológicos sustentaron la propuesta de validar a los poseedores del conocimiento andino-amazónico en un contexto universitario y de ejecutar el Diplomado en Biodiversidad y Diálogo de saberes. El curso se concibió como un programa semipresencial, dirigido a la práctica transdisciplinaria del diálogo de saberes relacionados con la diversidad biológica y cultural.

Uno de los objetivos principales fue una indagación compleja, transdisciplinaria, fenomenológica y hermenéutica de la educación que vincule a diferentes actores sociales y niveles de realidad (local, nacional y global) para construir una comunidad epistémica 
que contribuya a una educación intercultural y comunitaria, tanto a nivel urbano como rural. A nivel local, busca fortalecer las comunidades indígenas y los grupos comunales de afirmación cultural que existen en el área para hacer visible el conocimiento indígena. Validó el conocimiento tradicional y fortaleció a los líderes de la comunidad indígena Quechua-Lamas que asistieron al diplomado y, en general, a todos los participantes del diplomado, a quienes se les proveyó una visión intercultural. A nivel nacional, más allá de la experiencia del diplomado, se consolidó un grupo de trabajo sobre el tema de la innovación educativa en la Universidad Ricardo Palma.

A nivel global, se fomentó la creación de redes y el intercambio de mejores prácticas. Por ejemplo, se contribuyó en los debates en algunos eventos organizados por la Deutsche Gesellschaft für Internationale Zusammenarbeit (GIZ GmbH), el Programa de las Naciones Unidas para el Medio Ambiente (PNUMA) y la Universidad de las Naciones Unidas (UNU), entre otros.

El diplomado ayudó a los líderes indígenas a pasar de la transmisión oral de experiencias a la comunicación basada en texto. Además, en base a su experiencia y reflexión sistemática a lo largo del diplomado, los líderes de las comunidades indígenas redactaron algunas propuestas para resolver los desafíos locales y, en consecuencia, para empoderar a su comunidad.

Con el fin de evaluar a los indígenas que podrían contribuir como instructores en el diplomado y a los jóvenes líderes indígenas que podrían participar como estudiantes, se estableció un consejo académico. Este estaba compuesto por especialistas en la cultura andino-amazónica y la región San Martín con títulos universitarios y trabajos previos con los grupos comunitarios de afirmación cultural en el área. En el transcurso de dos años, entre las comunidades indígenas visitadas, se identificaron líderes y portadores de conocimientos tradicionales (reconocidos como tales por sus comunidades). Los evaluados y aceptados fueron incluidos en el diplomado. Esta fue la primera iniciativa de este tipo en el Perú.

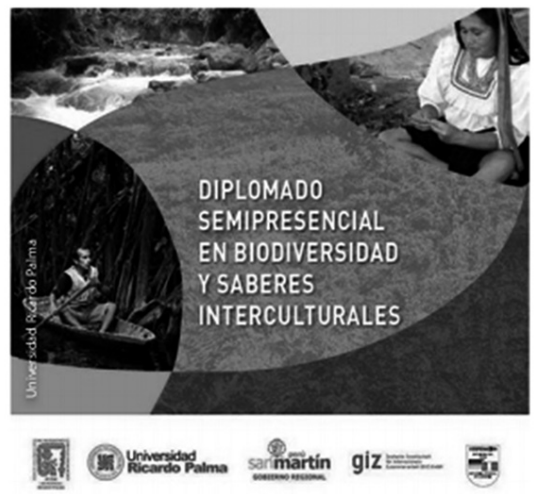

Presentación del diplomado 


\section{El diplomado en biodiversidad y saberes interculturales}

En Perú, los esfuerzos se han realizado para mejorar la educación comunitaria intercultural. Como se mencionó anteriormente, en la mayoría de los casos, estos esfuerzos están dirigidos a áreas rurales, y la educación aún está orientada hacia las costumbres y el conocimiento occidental. Muchos de los cursos dirigidos a las comunidades indígenas los sacan de su entorno, los llevan a la ciudad para capacitarlos en la cosmovisión occidental y los certifican, no obstante, en cursos que muchas veces no son apropiados para ellos. Se contó con el apoyo de la organización Waman Wasi.

En cambio, este proyecto se diferencia en esencia. Como punto de partida, considera que la cosmovisión amazónica es tan importante como la occidental. En el marco de un programa académico, ofrece un diálogo de saberes desarrollados en las comunidades andino-amazónicas. Su objetivo es comunicar a la nueva generación la pertinencia de los conocimientos tradicionales, la importancia de la vinculación urbana y rural, y una mejor integración social para el cuidado de la biodiversidad y la sostenibilidad.

El diplomado de aprendizaje mutuo sobre biodiversidad y diálogo de saberes surge del reconocimiento de la variedad biológica y cultural de la región andino-amazónica, y del papel crucial que desempeñan las instituciones regionales y las comunidades locales en su preservación y desarrollo. La diversidad muestra la milenaria tradición cultural en los colectivos indígenas y locales, y motiva el interés del Estado peruano y los gobiernos regionales para promover los intereses consagrados en el Convenio sobre la Diversidad Biológica (CBD), en la Ley General de Educación del Perú y en los diversos programas y políticas de conservación de la diversidad biológica.

El diplomado combina reflexiones teóricas con las metodologías de gestión de la diversidad cultural y biológica asociadas con la regeneración de activos culturales y naturales regionales. Este diplomado fue dirigido por profesores de la Universidad Ricardo Palma con contribuciones iguales de los poseedores del conocimiento local, es decir, hombres y mujeres sabios indígenas y líderes que compartirían con estudiantes del diplomado su conocimiento de algunos aspectos del bosque, agua, agricultura y medicina.

\section{Teorías de la metamorfosis: hipótesis de intervención}

Las dimensiones de las intervenciones que ofrece el diplomado son la conceptual (filosófica y epistemológica), la cultural (relevancia y contextualización), la tecnológica y la económica. La primera incluye la razón de la intervención y los paradigmas educativos que fundamentan las teorías de metamorfosis (extensión, cambio y transformación) que subyacen a todas las intervenciones educativas. La segunda considera asuntos relacionados con la relevancia de la intervención, en particular, sus efectos e impactos en 
diferentes actores y grupos culturales, y su percepción de los objetivos de la intervención. La tercera se refiere a la práctica del enfoque, la estrategia y los procedimientos, así como a la organización de los recursos disponibles para la intervención. La dimensión económica tiene que ver con los mecanismos de gestión y la planificación de recursos financieros que hacen posible esta intervención (Salinas, Ishizawa y Trellez 2014).

IPCEM y RCE Lima-Callao concibieron su intervención a partir de una visión de la metamorfosis del «campo» regional. Esto favoreció la participación de las comunidades indígenas, los profesores del curso del diplomado, así como los participantes y los profesores de otras instituciones, que no eran necesariamente locales.

La combinación de las dos tradiciones de conocimiento ha sido adoptada por el CBD desde 1993. Las iniciativas globales que siguieron, como la Evaluación de los Ecosistemas del Milenio (MEA) y la Evaluación Internacional de Ciencia y Tecnología Agrícolas para el Desarrollo (IAASTD) más reciente, reconocieron el valor de las comunidades indígenas, y los conocimientos locales y tradicionales. En lugar de una teoría del cambio completa, explícita o implícita, que podría esperarse de una intervención como el diplomado, la del IPCEM se basó en la convicción de la pertinencia del pensamiento complejo de Edgar Morin para la región de San Martín.

La viabilidad del diplomado tal como se diseńó radica en la existencia de (a) una documentación detallada del conocimiento sobre la rica biodiversidad de las comunidades indígenas en la región (especialmente del Quechua-Lamas), debido al trabajo de las instituciones de la sociedad civil y cooperación internacional como la GIZ; (b) maestros locales capaces de fomentar el diálogo de saberes intercultural; y (c) consenso sobre la necesidad de una plataforma para la comunidad epistémica regional que haga realidad el diálogo intercultural para reunirse, discutir y diseñar la formación de nuevos miembros. Sobre la base de estas fortalezas regionales, se implementó el Diplomado en Biodiversidad y Saberes Intercultural. (Salinas, Ishizawa y Trellez 2014)

\section{Diseño del curso}

El objetivo del diplomado ha sido equipar a los líderes de la comunidad, maestros, profesores de escuela, tecnólogos y profesionales de instituciones públicas y privadas, gobiernos regionales, estados y regiones andinas con una visión intercultural que comprenda y valore los conocimientos académicos y ancestrales relacionados a la regeneración y uso sostenible de la biodiversidad (IPCEM, GIZ GmbH 2012).

Al finalizar el diplomado, se espera que los graduados hayan logrado los siguientes objetivos:

a) Alcanzar una mayor conciencia sobre el cuidado de la biodiversidad a nivel local y global, teniendo en cuenta las diferentes opiniones sobre cómo abordar este problema 
b) Desarrollar habilidades para escuchar, comprender, y articular puntos de vista y posiciones de diversos actores del desarrollo

c) Adquirir competencia para la conversación e intercambio intercultural

d) Desarrollar la capacidad para elaborar enfoques interculturales de los problemas locales

e) Aplicar el aprendizaje del diplomado para trabajar en un tema local pertinente y presentar una propuesta de solución en forma de una monografía

El diseño e implementación del diplomado fue un proceso interactivo y en evolución. Fue iniciado por un consejo asesor que trabajó durante dos años con diferentes partes interesadas regionales (entre ellas, las comunidades indígenas Quechua-Lamas) para desarrollar la propuesta del diplomado y validar a sus participantes indígenas como instructores o estudiantes.

Este diplomado incluyó intercambios con científicos y trabajo de campo con Quechua-Lamas de comunidades indígenas que tenían antecedentes en afirmación cultural. Los módulos virtuales proporcionaron espacio para el autoaprendizaje con el apoyo en equipo de instructores virtuales/profesores/tutores. La tecnología de aprendizaje electrónico se utilizó en módulos de aprendizaje a distancia y se adaptó a la situación específica de cada estudiante.

La implementación fue un proceso de «aprender haciendo» que se validó en la vida cotidiana de los estudiantes. En un escenario altamente relacionado con los desafíos regionales, se establecieron dos visiones del mundo (dos formas de construir conocimiento). De un lado, a los estudiantes con una sólida visión del mundo occidental les gusta usar un lenguaje lineal y un marco lógico para la investigación. Del otro lado, estaban los estudiantes y líderes indígenas, cuyo conocimiento proviene, en gran medida, de conversaciones con la naturaleza, que comienzan a partir de sus experiencias comunitarias sobre cómo criar el campo (chacra en idioma quechua) e interactuar con el bosque o el agua, en relación con la luna.

La monografía de los estudiantes, una propuesta escrita para resolver un problema local basado en la experiencia de los estudiantes y la reflexión sistemática a lo largo del programa, es el resultado tangible del diplomado.

\section{Proceso de aprendizaje}

La metodología didáctica se basa en el reflejo de la propia experiencia. En un ambiente de confianza, tolerancia y respeto mutuo, uno es estimulado a dialogar con los demás sobre su visión del mundo, su percepción de la vida y la forma de construir el conocimiento. Gaston Pineaud lo manifiesta de la siguiente manera: 
Lo que queremos es cambiar la elección del conocimiento de una didáctica programada a una didáctica reflexiva y a una organización de reflexividad basada en la propia experiencia y en los desafíos concretos. Porque la reflexividad sobre las propias experiencias nos pone desde el principio dentro de la complejidad de la vida (Pineaud 2014, 40).

El trabajo de los participantes fue evaluado continuamente a través del proceso. En las fases presenciales, consistió en la evaluación de cada tema leyendo los resúmenes y desarrollándolos. En las fases de aprendizaje a distancia, se calificaron los informes de conferencias y la participación en foros y discusiones. Todas estas calificaciones juntas tienen un peso equivalente al de la calificación de la monografía.

\section{Resultados y debate}

Los interesados pasaron de las palabras a la acción. Finalmente, tecnólogos, profesionales, sabios de las comunidades indígenas y algunas organizaciones y agencias de cooperación internacional se conectaron a nivel local, nacional y global. Esto contribuyó a la creación de redes multiculturales y fortaleció el diplomado. IPCEM y la Universidad Ricardo Palma fueron pioneros en el enfoque de facilitar que líderes indígenas sin calificaciones académicas interactúen con los académicos calificados. Fue un gran desafío cambiar y abrir nuevos horizontes a la relación entre la educación superior y la sociedad para generar conocimiento pertinente.

Si bien existe abundante literatura sobre la biodiversidad desde una perspectiva académica, hay poco desde una perspectiva indígena. Por este motivo, tuvo que hacerse un esfuerzo especial para diseñar el plan curricular y los marcos metodológicos.

Los portadores de conocimientos tradicionales seleccionados por el Consejo Académico fueron reconocidos por la Universidad Ricardo Palma como profesores o como estudiantes en el diplomado. Además, los participantes indígenas que completaron exitosamente sus monografías obtuvieron certificados. Dado que esta fue la primera iniciativa de este tipo en el Perú, uno de los principales desafíos del curso fue superar la brecha entre la oralidad y la textualidad de los participantes indígenas, como lo revela su dificultad para escribir las monografías del diplomado.

Si bien en esta primera experiencia fue difícil alcanzar el completo potencial del diálogo de saberes, las bases del diplomado se sentaron con respecto al compromiso entre la comunidad académica y la comunidad indígena Quechua-Lamas.

GIZ GmbH, PNUMA y la Universidad Ricardo Palma financiaron el diplomado y ofrecieron algunas becas para estudiantes indígenas. Como los participantes eran muy pobres y venían de muy lejos, el diplomado debió proporcionarles, además, vivienda y alimentos. Asimismo, el compromiso con otras instituciones en el área donde se desa- 
rrolló el diplomado alentó que surja un trabajo basado en la solidaridad y la confianza mutua.

Se debe recordar que este diplomado introdujo a jóvenes participantes indígenas de la región en el uso de las TIC y el aula virtual. Esto, sin embargo, aumentó la duración programada del curso en un 30\%. Haber alcanzado competencia en TIC se evidenció cuando varios estudiantes del diplomado participaron en la Conferencia Iberoamericana de Educación Ambiental celebrada en Lima en septiembre de 2014, donde presentaron sus monografías finales. Los participantes valoraron esta oportunidad de comunicarse a nivel local, nacional y global.

La paridad de conocimiento de los estudiantes indígenas y maestros fue reconocida y apreciada cuando participaron en la Segunda Conferencia Regional de América del RCE celebrada en Lima en febrero de 2013, lo cual fue una oportunidad para la incorporación de su trabajo a nivel global.

\section{Conclusiones}

1) Este proyecto exploró nuevos horizontes en los marcos epistemológicos de la Educación Superior. Las conclusiones alcanzadas invitan a mirar hacia nuestros orígenes para encontrar nuestra historia, a mirar nuestros ecosistemas para encontrar sus características, y a mirar en la cosmovisión andina amazónica como bases para contribuir con la EDS para desarrollar prácticas que apoyen vidas dignas.

Están surgiendo formas de pensar que cuestionan los fundamentos mismos del conocimiento dominante, y están abriendo nuevos marcos epistémicos para la construcción del conocimiento y la comprensión de los fenómenos dinámicos, complejos, naturales y sociales que subyacen en la existencia humana. Estas nuevas formas de pensamiento y creación de conocimiento tienen el potencial de abordar, con pertinencia, los desafíos que enfrentan la educación y la humanidad para construir sociedades más sostenibles.

2) La EDS enfrenta el desafío fundamental de transformar la conciencia: la forma en que las personas piensan y sienten el mundo. Es necesario reemplazar un razonamiento instrumental y mercantilista por uno ético y humanista que nos permita recuperar el significado de la vida, la condición humana y el hecho de estar en el mundo. Los seres humanos deben entender que viven en un planeta que no es ilimitado y cuyas dinámicas obedecen a procesos complejos e irreversibles que no pueden controlar.

3) Como mínimo, la educación para una vida sostenible requiere tener en cuenta los desafíos formativos y deformantes de la vida cotidiana, a nivel local, nacional y mundial. Esta es una condición básica para transformar la educación y sacarla de 
su abstracción. En el proceso educativo, formal, informal y no formal, uno debe cuestionarse cómo se construye el conocimiento y cómo este elabora estructuras mentales.

4) Es necesario cambiar los marcos filosóficos, epistemológicos y didácticos de la educación para cambiar la visión reduccionista, fragmentada y determinista del sistema educativo y ampliar las funciones universitarias. Esto podría hacerse examinando diferentes escenarios futuros; proporcionando a la universidad una visión intercultural; y gestionando diversos sistemas de conocimiento, incluido el local.

5) Se construyó una comunidad epistémica local-global para fomentar la transdisciplinariedad en el diálogo de saberes con el fin de crear espacios de paz, cooperación y confianza, y generar visiones compartidas que puedan resolver los principales desafíos locales, incluidos aquellos en el área de la biodiversidad.

6) Para ser sostenibles, las iniciativas necesitan tiempo para madurar. Uno necesita entender la región donde se lleva a cabo la experiencia práctica, buscar oportunidades para su inserción en el campo académico y en los debates ecológicos nacionales, y valorar su anclaje local y global. Tales iniciativas deben estar arraigadas en la vida institucional, en las organizaciones indígenas y populares, y en la sociedad civil.

7) El desarrollo de esta práctica generó un debate sobre la relevancia y el valor del sistema de conocimiento andino-amazónico. Además, estableció un importante precedente para su valoración en el sistema de educación superior peruano. Se generaron sinergias con otras actividades educativas, en particular un curso de Ingeniería Hidráulica que a se investiga en la Facultad de Ingeniería/Escuela Profesional de Ingeniería Civil de la Universidad Ricardo Palma.

8) Se realizó una investigación compleja, transdisciplinaria, fenomenológica y hermenéutica con buena interacción entre diferentes niveles de realidad. A nivel local, el conocimiento tradicional se fortaleció y el liderazgo de las comunidades indígenas se le formó desde una visión intercultural. A nivel nacional, se consolidó un grupo de trabajo sobre educación e innovación en la Universidad Ricardo Palma y se desarrolló la propuesta Ayni». A nivel mundial, se alentó el intercambio y la creación de redes, que contribuyen a los debates y permiten compartir mejores prácticas en congresos.

9) El Propuesta Ayni explora una visión dinámica, compleja y transdisciplinaria de la educación. Se basa en un pensamiento circular multidireccional y multireferencial que considera procesos, agentes, organizaciones e interacciones de los sistemas naturales y sociales que desempeñan un papel en el proceso educativo. Promueve un enfoque intercultural e incluye el diálogo de saberes, especialmente, en el sistema andino-amazónico. Busca unir el arte y la ciencia para humanizar el conocimiento. Se trata de crear una visión compartida de retroalimentación positiva para desa- 
rrollar planes de estudio transdisciplinarios desde perspectivas locales, nacionales y globales para brindar calidad y pertinencia a la EDS.

10) La educación de los niños indígenas y los jóvenes indígenas debe comenzar con el reconocimiento de la validez de su forma de pensar y de estar en el mundo. Debe convertirse en un espacio para aprender sus conocimientos tradicionales y su sabiduría, y para fortalecer sus idiomas maternos y las prácticas campesinas que aumentan la biodiversidad local, lo que puede servirles como una fuente de empleo. De esa manera, garantizan su alimentación, salud y preservación de su cultura. La educación también debe ser un espacio para acceder al conocimiento occidental a través de la lectura y escritura, tanto en español como en otros idiomas. De igual forma, pueden aprovechar el uso de tecnologías de la información. Debería ser una reunión de dos culturas, que se respeten mutuamente y que continuamente tengan un diálogo de conocimiento.

\section{Reconocimientos}

Esta experiencia fue posible gracias a la convocatoria de propuestas de GIZ GmbH, a través de su Programa de Desarrollo Rural Sostenible (PDRS); a la URP, por su compromiso para promover este proyecto innovador; y a GIZ GmbH, el PNUMA y la URP por su apoyo financiero. La autora también desea reconocer la solidaridad del consejo académico que unió a varias organizaciones regionales, y a Wamanwasi, las comunidades indígenas Quechua-Lamas que nos acogieron y abrigaron en esos caminos de luz y esperanza con su amor y sabiduría. Finalmente, se agradece al Instituto para el Estudio Avanzado de la Sostenibilidad de la Universidad de las Naciones Unidas (UNU-IAS), que nos apoyó y reconoció nuestra experiencia con el RCE Award for Outstanding Flagship Project en la 9a Conferencia Mundial de RCE celebrada en 2014 en Okayama, Japón, y, por lo tanto, nos ayuda a hacer visible nuestro trabajo, a la vez que fortalece nuestro desarrollo. 


\section{Referencias}

Fadeeva, Z., Payyappallimana, U., Tabucanon, M. and Chhokar K. B. eds. (2014). Building a Resilient Future through Multistakeholder Learning and Action: Ten Years of Regional Centres of Expertise on Education for Sustainable Development. Tokyo: United Nations University Institute for the Advanced Study of Sustainability. IPCEM, GIZ GmbH. 2012.

Foundations of Diploma Course on Biodiversity and Dialogue of Knowledge Systems. GIZ GmbH, Universidad Ricardo Palma.

Ishizawa, J. (2009). Espistemologías de la educación intercultural. Memorias del 2do taller sobre Educación Intercultural y Epistemologías Emergentes. Lima: PRATEC.

Lajo, J. (2005). Qhapaq Nan, la ruta Inka de sabiduría. Lima: Editorial Amaru Runa-CENES. Maturana, H. (2014). Transformación en la convivencia. Argentina: Ediciones Granica.

Maldonado, C. E. (Ed.) (2011). Complejidad: Revolución científica ...

Prigogine, I. and Stengers, I. (1983). La Nueva Alianza: Metamorfosis de la ciencia. Alianza Editorial.

Quijano, A. (2015). Cuestiones y horizontes. CLACSO.

Ramos, G. (1992). La Universidad Peruana en el siglo XXI. Lima: Universidad Ricardo Palma.

Rengifo, G. (2003). La enseñanza es estar contento. Educación y afirmación cultural andina. Lima: PRATEC.

SAlinAs, T. (2009). Alternativas para una propuesta metodológica de autoevaluación de la calidad y pertinencia de la formación profesional de ingenieros. En La educación en Ciencias e Ingeniería. Calidad, innovación pedagógica y cultura digital, 108-109. España: Universidad de Alcalá.

Salinas, T., Ishizawa, J. and Trellez, E. (2014). Systematization of the Diploma Course in Biodiversity and Dialogue of Knowledge Systems. IPCEM - Universidad Ricardo Palma.

Vallejo-Gómez, N. (2009). Morin, humanista planetario. Lima: Fondo Editorial Derrama Magisterial. 


\section{Apéndice A}

\section{Leyenda para entender la propuesta «Ayni»}

\section{NIVEL I (Local) Nivel de la Universidad}

\begin{tabular}{|l|l|}
\hline Nodo A & $\begin{array}{l}\text { Conexiones: equipo de sistemas de información, diagnósticos y estudios futuros a nivel } \\
\text { local, nacional y global. } \\
\text { Interacciones: con el Nivel I, Nivel II, Nivel III, los nodos B y C, y crea visiones com- } \\
\text { partidas. } \\
\text { Proporciona: información relevante y actualizada para el desarrollo y ajuste de planes de } \\
\text { estudio, y para la generación pertinente de visiones compartidas. } \\
\text { Los signos positivos implican una retroalimentación creciente de los procesos; los nega- } \\
\text { tivos, la disminución de la calidad de la educación superior. }\end{array}$ \\
\hline Nodo B & $\begin{array}{l}\text { Conexiones: marcos epistemológicos, didácticos, psicológicos y diseńo curricular. } \\
\text { Interacciones: con Nodo A, Nodo C, Nivel II y Nivel III, y crea visiones compartidas. } \\
\text { Proporciona: currículo, planes de estudio, syllabus, ejecución de programas de pregrado } \\
\text { ycrea visiones compartidas. }\end{array}$ \\
\hline Nodo C & $\begin{array}{l}\text { Conexiones: Investigación, innovación, patentes, programas de posgrado y gestión del } \\
\text { conocimiento. } \\
\text { Interacciones: con Nodo A, Nodo B, Nivel II y Nivel III y crea visiones compartidas. } \\
\text { Proporciona: líneas de investigación para la innovación, cursos de postgrado, equipos } \\
\text { de investigación, sistemas de gestión del conocimiento, patentes y visiones compartidas } \\
\text { para la Educación para el Desarrollo Sostenible (EDS). }\end{array}$ \\
\hline
\end{tabular}

\section{NIVEL II (Nacional)}

Conexiones: enlaces universitarios y niveles de educación básica para comprender y resolver las deficiencias de los estudiantes que están comenzando programas universitarios. Enlaces universitarios y diferentes saberes (especialmente con relación a los conocimientos tradicionales y populares).

Vincula la universidad y el Sistema Nacional de Ciencia y Tecnología, los sistemas de producción (privados y públicos) con políticas para estudios, y la investigación y gestión del conocimiento. Asimismo, relaciona la universidad y los sistemas de educación formal e informal para el Desarrollo Sostenible (DS).

Interacciones: espacios transdisciplinarios de diálogo, una mayor comprensión de los problemas y fenómenos naturales y sociales.

Proporciona: información de contexto para mejorar la calidad y pertinencia de la educación, visiones compartidas de país. 


\section{NIVEL III (Global)}

Conexiones: relaciones con otras universidades dentro de la región y alrededor del mundo.

Interacciones: con expertos de otras universidades, agencias de cooperación internacional, UNU, UNESCO, GIZ GmbH, entre otros.

Proporciona: información para guiar a los académicos y el trabajo de investigación, a fin de avanzar en el conocimiento global. Trabaja sobre una base de ciudadanía planetaria para lograr Objetivos de Desarrollo Sostenible (ODS). 


\section{Apéndice B}

\section{Módulos del diplomado}

\begin{tabular}{|c|c|c|}
\hline Módulo I & $\begin{array}{l}\text { Biodiversidad y Diálogo de } \\
\text { Saberes }\end{array}$ & $\begin{array}{l}\text { Este módulo presenta el diplomado; establece } \\
\text { los fundamentos conceptuales para abordar la } \\
\text { diversidad, tanto biológica como cultural, y el diálogo } \\
\text { transdisciplinario de saberes; y se enfoca en su base } \\
\text { operativa a través de capacitación en tecnologías de la } \\
\text { información y comunicación. Incluye una visita a las } \\
\text { comunidades indígenas locales Quechua-Lamas para } \\
\text { observar los conocimientos tradicionales en la práctica. }\end{array}$ \\
\hline Módulo II & Ecología y Ecologías & $\begin{array}{l}\text { Partiendo del Módulo I, el segundo introduce la ecología } \\
\text { como marco conceptual para un diálogo de saberes. Se } \\
\text { trata de comprender todo el ecosistema y cada una de } \\
\text { sus etapas -chacra, bosque y agua- desde la visión } \\
\text { tecnocientífica y desde el conocimiento local de una } \\
\text { manera integrada. }\end{array}$ \\
\hline Módulo III & $\begin{array}{l}\text { Enfoques Interculturales, } \\
\text { Género, Salud y Soberanía } \\
\text { Alimenticia }\end{array}$ & $\begin{array}{l}\text { Se estudia el uso de la categoría "género" en las políticas, } \\
\text { y las prácticas de alimentación y salud en el ámbito } \\
\text { científico. Se analiza, también, desde la visión de las } \\
\text { comunidades indígenas. }\end{array}$ \\
\hline Módulo IV & $\begin{array}{l}\text { Biodiversidad y Cambio } \\
\text { Climático }\end{array}$ & $\begin{array}{l}\text { El tema central del Módulo IV es la respuesta local } \\
\text { dirigida a la regeneración de biodiversidad en el } \\
\text { contexto del cambio climático tanto para adaptación } \\
\text { como mitigación. }\end{array}$ \\
\hline Módulo V & $\begin{array}{l}\text { Desarrollo Sostenible y } \\
\text { Biocomercio }\end{array}$ & $\begin{array}{l}\text { El módulo } \mathrm{V} \text { está dirigido a debatir formas de mejorar } \\
\text { las condiciones de vida y los medios de vida de las } \\
\text { comunidades, y promueve un diálogo de saberes y } \\
\text { cosmovisiones al centrarse en la convivencia pacífica. } \\
\text { Así mismo, se centra en las respuestas locales de las } \\
\text { comunidades. }\end{array}$ \\
\hline Módulo VI & $\begin{array}{l}\text { Pueblos indígenas, } \\
\text { Conservación de la } \\
\text { Biodiversidad y Legislación } \\
\text { Medioambiental }\end{array}$ & $\begin{array}{l}\text { Se observa la visión de los pueblos indígenas y las } \\
\text { comunidades locales sobre la regeneración de la } \\
\text { biodiversidad en un contexto global. }\end{array}$ \\
\hline $\begin{array}{l}\text { Módulo } \\
\text { VII }\end{array}$ & Seminario de Investigación & $\begin{array}{l}\text { Prepara a los estudiantes para desarrollar sus monografías, } \\
\text { que son propuestas para resolver problemas locales, y } \\
\text { que están basadas en la experiencia de los estudiantes } \\
\text { y la reflexión sistemática a lo largo del programa. Es el } \\
\text { resultado tangible del diplomado. }\end{array}$ \\
\hline
\end{tabular}

